\title{
Achieving Arbitrary Multiplexing Rates for MIMO-OFDM Systems by Hybrid Cyclic Delay Diversity
}

\author{
Hsien-Wen Chang, Member, IEEE, and Li-Chun Wang, Fellow, IEEE
}

\begin{abstract}
This paper proposes a new hybrid cyclic delay diversity (HCDD) scheme for multiple input multiple output (MIMO) orthogonal frequency division multiplexing (OFDM) systems. Unlike the existing double space time transmit diversity (DSTTD) and stacked cyclic delay diversity (SCDD) schemes that are suitable for integer multiplexing rates, the proposed HCDD scheme can achieve arbitrary non-integer multiplexing rates. Our results demonstrate that the HCDD scheme can provide flexibility in selecting the required number of transmitting antennas and in adjusting diversity and multiplexing gains to match various users requirements. The advantages of the proposed $\mathrm{HCDD}$, including the flexible rate assignment and less complex receivers, are presented in applications of scalable video broadcasting (SVB).
\end{abstract}

Index Terms-MIMO-OFDM, DSTTD, CDD, SCDD, diversitymultiplexing-tradeoff.

\section{INTRODUCTION}

$\mathbf{M}$ ULTIPLE-INPUT multiple-output (MIMO) antenna techniques can improve system capacity, enhance link reliability, and reduce interference. Improvements to system capacity and link reliability in MIMO systems result from the spatial multiplexing (SM) gain and the diversity gain, respectively. Spatial multiplexing gain is contributed by the parallel transmissions of independent data streams in the multiple pairs of transmitting and receiving antennas. In contrast, multiple replicas of the same signal in multiple antennas yield spatial diversity gain, and thus improve link reliability. Thus, the performance of MIMO antenna systems should be designed based on the multiplexing and diversity tradeoff [1].

Another important broadband wireless transmission technique is orthogonal frequency division multiplexing (OFDM), which can overcome the inter-symbol interference (ISI) when transmitting high-rate data in a frequency selective fading channel. Furthermore, OFDM can also provide a degree of freedom in subcarriers for resource allocation. Because MIMO and OFDM can enhance system performance from different aspects, MIMO-OFDM has become an important research area in the past decade [2], [3]. Thus, a natural question for MIMO-OFDM systems may be raised as follows: Can

Manuscript received April 7, 2012; revised September 10 and November 26, 2012. The associate editor coordinating the review of this paper and approving it for publication was R. Dinis.

H.-W. Chang is with the Information and Communications Research Laboratories, Industrial Technology Research Institute, Hsinchu, Taiwan.

L.-C. Wang is with the Department of Electrical and Computer Engineering, National Chiao Tung University, Hsinchu, Taiwan (e-mail: lichun@cc.nctu.edu.tw).

This work is sponsored by National Science Council, Taiwan, under the contract NSC-100-2221-E-009-103-MY3.

Digital Object Identifier 10.1109/TCOMM.2013.020413.120239 the diversity and multiplexing tradeoff in MIMO systems be achieved differently from MIMO-OFDM if OFDM provides another degree of freedom in subcarrier?

In the literature, several MIMO-OFDM systems have been proposed to achieve diversity and multiplexing gains. In [4], a double space time transmit diversity (DSTTD) transmission architecture was proposed for MIMO-OFDM systems, decoded with a pre-whitening filter followed by a minimum-Euclideandistance decoder. In [5], three types of group receivers were proposed to separate the filtered multiplexing streams, followed by a space-time decoder. In [6] switching between two hybrid MIMO structures was proposed to improve transmission over wireless systems. An algorithm to find the optimal antenna grouping configuration maximizing the throughput of the diversity-multiplexing combined system was proposed in [7]. The authors in [8] presented a three-step cancelling mechanism to separate two Alamouti units in DSTTD systems. However, the existing MIMO-OFDM architectures can only achieve the integer multiplexing rate.

Cyclic delay diversity (CDD) [9] is another popular diversity technique that has been proposed for MIMO-OFDM systems. With CDD, the same OFDM signal is transmitted over different antennas, each of which experiences different cyclic shifts. Thus, extra frequency selectivity can be created at the receiver without changing the receiver design [9]. Combining CDD with the SM-based MIMO can provide both the diversity and multiplexing gains. The diversity-multiplexing tradeoff (DMT) analysis of MIMO systems with stackedCDD (SCDD) and their capacity were reported in [10] and [11], respectively. The combination of CDD and SM-based MIMO systems was adopted by [12] without considering the non-integer multiplexing rates. In our previous work [13], we proposed a hybrid scheme to provide non-integer multiplexing rates by combining SCDD and SM-based MIMO-OFDM systems. However, the systematic code construction and the outage capacity performance have not yet been reported.

The objective of this paper is to investigate how a MIMOOFDM system can flexibly exploit the diversity and multiplexing gains to support various data rates and link reliability requirements. The proposed hybrid cyclic delay diversity (HCDD) scheme is designed by combining pure CDD and spatial multiplexing in the OFDM systems. By taking advantage of the subcarrier rate assignment in OFDM systems, the proposed HCDD scheme can achieve non-integer multiplexing rates. A novel systematic transmission architecture is proposed to achieve flexible diversity-multiplexing-tradeoff in MIMO- 
OFDM systems. Thus, in terms of achieving tradeoff between multiplexing rates and diversity, the proposed HCDD is more flexible than the existing MIMO-OFDM systems with CDD. The corresponding decoder architecture, theoretical system capacity and the impact of antenna correlation are also presented. To demonstrate the advantages of HCDD, we apply the HCDD scheme to scalable video broadcasting (SVB) .

The rest of this paper is organized as follows. Section II reviews the background of CDD and the SCDD scheme. The cyclic delay values and achieved diversity gain are also analysed. In Section III, we present the rate-flexible HCDD scheme for MIMO-OFDM systems and the systematic construction and detection method for HCDD, followed by the application of HCDD to SVB. In Section IV, numerical results are given to show that HCDD can achieve any multiplexing and diversity tradeoff to fill the performance gap of the other existing schemes in terms of outage capacity and bit error rate. The simulation results of SVB are also presented. Our concluding remarks are given in Section V.

\section{REVIEW OF CDD AND SCDD}

\section{A. $C D D$}

In OFDM systems, the information-bearing symbols $s[k]$ are modulated onto orthogonal subcarriers via Inverse Fast Fourier Transform (IFFT) operation. The received signals can be simply represented on a per-tone basis as $y_{k}=H_{k} s_{k}$, where $H_{k}$ is the single-input single-output (SISO) channel frequency response. For simplicity, additive white Gaussian noise (AWGN) is ignored.

When multiple transmit antennas are available, artificial frequency selectivity can be created by transmitting cyclically shifted versions of the original signal. With $M_{t}$ transmitting antennas, the signal transmitted from the $m$-th antenna is written as

$x_{m}[n]=\frac{1}{\sqrt{N M_{t}}} \sum_{k=0}^{N-1} s_{k} \exp \left\{j \frac{2 \pi k\left(\left(n-\delta_{m}\right) \bmod N\right)}{N}\right\}$,

where $N$ is the number of subcarriers, and $\delta_{m}$ is the value of cyclic delay associated with the $m$-th antenna, $m=$ $1,2, \ldots, M_{t}$. The cyclically shifted signal $x_{m}[n]$ can be also represented by the frequency-domain equivalent operation [9]:

$$
x_{m}[n]=\frac{1}{\sqrt{M_{t} N}} \sum_{k=0}^{N-1}\left(e^{-j \frac{2 \pi \delta_{m} k}{N}} s_{k}\right) e^{j \frac{2 \pi n k}{N}} .
$$

Letting $W_{N}=e^{-j \frac{2 \pi}{N}}$, the received per-tone signal from $M_{t}$ transmitting antennas can be represented by

$$
\begin{aligned}
y_{k}^{(C)} & =\frac{1}{\sqrt{M_{t}}}\left(\sum_{m=1}^{M_{t}} H_{m, k} W_{N}^{\delta_{m} k}\right) s_{k} \\
& =\frac{1}{\sqrt{M_{t}}} H_{k}^{(C)} s_{k},
\end{aligned}
$$

where $\mathbf{H}_{k}=\left[\begin{array}{lll}H_{1, k}, & \ldots & H_{M_{t}, k}\end{array}\right]$ is the multiple-input single-output (MISO) channel frequency response, and the composite channel characteristic $H_{k}^{(C)}=\sum_{m=1}^{M_{t}} H_{m, k} W_{N}^{\delta_{m} k}$. It is not necessary to change the design at the receiver as long as the composite channel characteristic $H_{k}^{(C)}$ can be tracked. Usually, forward error correction (FEC) techniques are implemented to exploit the frequency diversity gain provided by CDD [14].

\section{B. $S C D D$}

The CDD scheme can incorporate spatial multiplexing techniques by stacking multiple groups of CDD antennas, which is referred to as the stacked CDD [10] or the cyclic delay assisted SM-OFDM (CDA-SM-OFDM) [11]. The basic idea of SCDD is to separate data streams into the different groups upon which the CDD scheme is implemented.

Consider a single-user MIMO-OFDM system with $M_{t}$ transmitting antennas, $M_{r}$ receiving antennas, and $N$ subcarriers. Let $r$ be the number of streams to be transmitted, and $\mathbf{s}_{k}=\left[s_{k}^{(1)}, \ldots, s_{k}^{(r)}\right]^{T}$ be the symbols of each stream at the $k$-th subcarrier after channel coding and quadrature amplitude modulation (QAM). $M_{t}$ transmitting antennas are divided to $r$ groups, each of which is equipped with $B=M_{t} / r$ antennas. In addition, $\delta_{m}$ is the value of cyclic shift associated with the $m$-th antenna. The received signal at the $k$-th subcarrier can be expressed as

$$
\mathbf{y}_{k}^{(S)}=\frac{1}{\sqrt{M_{t}}} \mathbf{H}_{k}^{(S)} \mathbf{s}_{k} .
$$

The $(i, j)$-th element of the composite channel $\mathbf{H}_{k}^{(S)}$ (for $i$ th receive antenna and $j$-th transmit antenna group) in (4) is given by

$$
\mathbf{H}_{i, j, k}^{(S)}=\sum_{m=(j-1) B+1}^{j B} H_{i, m, k} W_{N}^{\delta_{m} k},
$$

where $i \in\left\{1, \ldots, N_{r}\right\}, j \in\{1, \ldots, r\}$, and $H_{i, m, k}$ are the independent Rayleigh channel frequency responses at the $k$ th subcarrier from the $m$-th transmitting antenna to the $i$-th receiving antenna for $k=1$ to $N$.

In the following, the (multiplexing) rate of any transmission scheme is defined as the average number of QAM symbols transmitted per subcarrier. The rate for the SCDD scheme is exactly $r$.

\section{Cyclic Delay Value Analysis}

The diversity order provided by CDD depends on the cyclic delay values applied to the transmission antennas. In theory, with more independent paths received by the receiver, higher diversity gain is achieved. In a MISO system, suppose that the channel memory from each transmit antenna to the receiving antenna is known to the transmitter and denoted as $L_{i}, i \in$ $\left\{1, \ldots, M_{t}\right\}$; then the cyclic delay value of the $i$-th transmit antenna should be assigned as $\delta_{i}=\delta_{i-1}+L_{i-1}, i \in\left\{2, M_{t}\right\}$ $\left(\delta_{1}=0\right)$ according to the above principle. Cyclic delay values larger than $\left\{\delta_{i}\right\}$ do not further increase the diversity gain [15].

However, in open loop systems, the channel memory is usually unknown to the transmitter. Therefore, a large cyclic delay value is proposed as [16]

$$
\delta_{i}=\frac{N(i-1)}{M_{t}}=\frac{N}{M_{t}}+\delta_{i-1} .
$$


Using a large cyclic delay can decorrelate adjacent $M_{t}$ subcarriers, i.e.,

$$
E\left[H_{k}^{(c)}\left(H_{k+l}^{(c)}\right)^{*}\right]=0, \quad|l| \leq M_{t}-1,
$$

assuming the transmitting antennas are spatially uncorrelated and have identical power delay profile, and the coherent bandwidth is larger than $M_{t}$ subcarrier spacings. In other words, the spatial diversity from $M_{t}$ transmitting antennas is transformed into frequency diversity among $M_{t}$ adjacent subcarriers by the large cyclic delay.

The effect of the artificial frequency selectivity from a large delay CDD can be utilized in both frequency-flat and frequency-selective channels. In the frequency-selective fading channels, the coherent bandwidth $B_{c}$ is defined as the bandwidth over which the channel frequency response can be regarded unchanged. A general rule of thumb is that $B_{c} \approx 1 / \tau_{\max }$, where $\tau_{\max }$ is the maximum delay spread and is usually within $1 / 3$ of the guard interval (GI) of OFDM systems [17]. If GI is designed to be $1 / 8$ of the OFDM useful symbol time, then $B_{c}$ will cover more than 20 subcarriers. To take advantage of both artificial frequency selectivity from CDD and inherent frequency selectivity from broadband channel, the symbols of a stream can be allocated in a block-by-block manner. Each block consists of several adjacent subcarriers to get the benefits of the decorrelated channel caused by CDD, and different blocks are sufficiently separated to have the inherent frequency diversity of broadband channels.

\section{Proposed Hybrid CDD (HCDD) Scheme}

\section{A. Basic Concept}

In addition to diversity and multiplexing in the antenna spatial dimension, as exploited by the existing DSTTD and SCDD schemes, we propose a hybrid CDD scheme to explore the subcarrier frequency dimension in OFDM systems. Unlike most existing MIMO-OFDM schemes, in which all subcarriers transmit at the same rate, the proposed HCDD scheme can support different rates at each subcarrier or a group of subcarriers. Let $r_{k}$ be the number of streams assigned to the $k$-th subcarrier, and $\mathbf{s}_{k}=\left[s_{k}^{(1)}, \ldots, s_{k}^{\left(r_{k}\right)}\right]^{T}$. The received signal at the $k$-th subcarrier can be expressed as

$$
\begin{aligned}
\widetilde{\mathbf{y}}_{k} & =\frac{1}{\sqrt{M_{t}}} \widetilde{\mathbf{H}}_{k} \mathbf{s}_{k}+\mathbf{w}_{k} \\
& =\frac{1}{\sqrt{M_{t}}}\left(\mathbf{H}_{k} \mathbf{D}_{M_{t}, k} \mathbf{V}_{k}\right) \mathbf{s}_{k}+\mathbf{w}_{k},
\end{aligned}
$$

where $\mathbf{H}_{k}$ is the MIMO channel frequency response, and $\mathbf{D}_{M_{t}, k} \triangleq \operatorname{diag}\left\{W_{N}^{\delta_{1} k}, \ldots, W_{N}^{\delta_{M_{t}} k}\right\}$ is a diagonal matrix corresponding to the frequency equivalent operation of cyclic delay at each transmitting antenna. $\mathbf{V}_{k}=\mathbf{1}_{M_{t} / r_{k}} \otimes \mathbf{I}_{r_{k}}$ is used to evenly distribute $r_{k}$ symbols to $M_{t}$ antennas, where $\mathbf{1}_{M_{t} / r_{k}}$ is an all-one vector and $\otimes$ denotes Kronecker production. In addition, $\mathbf{w}_{k}$ is the AWGN vector with distribution $\mathrm{CN}\left(\mathbf{0}, N_{0} \mathbf{I}_{M_{r}}\right)$. The multiplexing rates of each subcarrier are carried out through the proposed design of the $\mathbf{V}_{k}$. For the special case $\mathbf{V}_{k}=\mathbf{1}_{M_{t}}$ (i.e. $r_{k}=1$ ), the same symbol is transmitted to several antennas to achieve diversity. $\mathbf{V}_{k}=\mathbf{I}_{M_{t}}$ (i.e. $r_{k}=M_{t}$ ) represents the case in which $M_{t}$ independent symbols are simultaneously transmitted to $M_{t}$ antennas to achieve multiplexing gain. For those antennas transmitting identical symbols, $\mathbf{D}_{M_{t}, k}$ provides diversity gain similar to CDD. For those antennas transmitting independent symbols, the phase rotation caused by $\mathbf{D}_{M_{t}, k}$ becomes part of the final channel response perceived by receiver.

One advantage of HCDD is that the rate of space-time codes can be adjusted flexibly according to the system requirements by assigning $r_{k}$ to different subcarriers. Furthermore, the number of transmitting antennas for HCDD can also be adjusted flexibly. The minimum number of transmitting antennas required for DSTTD or SCDD is four, while HCDD can be applied to systems with only two transmitting antennas.

\section{B. Systematic Construction of HCDD Codes}

From the general concept of HCDD, $r_{k}$ can be flexibly assigned to each subcarrier, and is only limited by the number of transmitting antennas $M_{t}$. However, since there are a large number of subcarriers in contemporary communications systems, the decoding process becomes extremely difficult to manage if $r_{k}$ is arbitrarily assigned without rules. To control the complexity of the encoding and decoding process and to maintain the flexibility of the HCDD scheme, the following systematic construction method is proposed.

Initially, all the subcarriers are divided to many equal-sized resource blocks, each of which has $N_{q}$ subcarriers. Then, $r_{k}$ associated with $N_{q}$ subcarriers in one resource block is managed. In our method, $M_{t}$ antennas can be equally divided into $r_{k}$ streams. Assuming that $M_{t}$ has $P$ factors $\left\{f_{1}, \ldots, f_{P}\right\}$, then $r_{k}$ must belong to the set $\left\{f_{1}, \ldots, f_{P}\right\}$. The rate of the HCDD code is determined by

$$
r=\frac{\sum_{p=1}^{P} f_{p} N_{p}}{N_{q}},
$$

where $N_{p}$ is the number of subcarriers with rate equal to $f_{p}$. If the target rate of HCDD is $r_{G}$, then $\left\{N_{1}, \ldots, N_{P}\right\}$ must satisfy the following constraints:

$$
\left\{\begin{array}{l}
f_{1} N_{1}+f_{2} N_{2}+\ldots+f_{P} N_{P}=r_{G} N_{q} \\
N_{1}+N_{2}+\ldots N_{P}=N_{q} \\
N_{p} \in \mathbb{Z}+, \quad \forall p .
\end{array}\right.
$$

The code construction can be implemented using the algorithm depicted in Fig. 1. The HCDD encoder chooses one feasible subcarrier number combination $\left[N_{1}, N_{2}, \ldots, N_{P}\right]$, which is applied to all resource blocks.

The proposed construction method has the following two advantages. First, since CDD is an open-loop transmitting diversity technique, no channel state information (CSI) is required at the transmitter. It is beneficial to apply $r_{k}$ uniformly among all the subcarriers. Secondly, as long as the finite possible combinations are clearly defined and indexed at both the transmitter and receiver, the decoder needs only the index number $\left[N_{1}, N_{2}, \ldots, N_{P}\right]$ to perform decoding, so much less information is needed than other construction methods.

\section{Detection of $H C D D$}

According to the construction method introduced in Section III-B, the decoding process of the proposed HCDD 


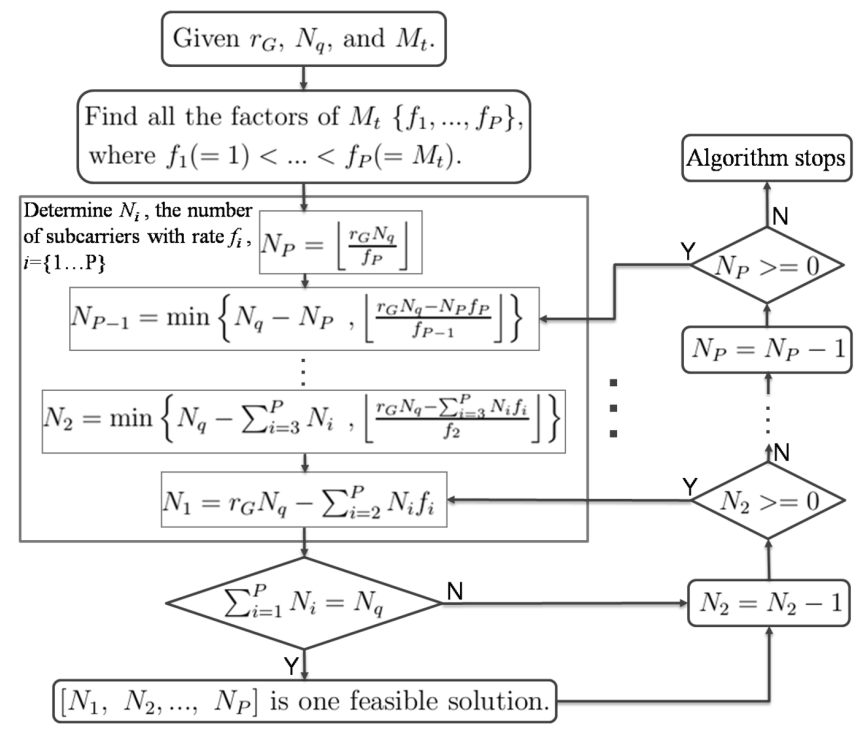

Fig. 1. Algorithm for HCDD code construction.

scheme also becomes very easy. If a receiver is informed of the subcarrier combination $\left[N_{1}, N_{2}, \ldots, N_{P}\right]$ chosen by transmitter, the multiplexing rate at each subcarrier $r_{k}$ is known. Then the decoding process is performed straightforwardly on a tone-by-tone basis.

For the subcarriers with $r_{k}=1, \widetilde{\mathbf{H}}_{k}$ is a $\left(M_{r} \times 1\right)$ vector, and scalar minimum-mean-square-error (MMSE) detector is given as:

$$
\mathbf{G}_{k}^{M M S E}=\left(\widetilde{\mathbf{H}}_{k}^{\mathcal{H}} \widetilde{\mathbf{H}}_{k}+\frac{1}{S N R}\right)^{-1} \widetilde{\mathbf{H}}_{k}
$$

For the subcarriers with $r_{k} \geq 2$, QR-decomposition (QRD) can be used to achieve successive interference cancellation (SIC). The QRD of $\widetilde{\mathbf{H}}_{k}$ is given by $\widetilde{\mathbf{H}}_{k}=\widetilde{\mathbf{Q}}_{k} \widetilde{\mathbf{R}}_{k}$, where $\widetilde{\mathbf{Q}}_{k}$ is the $\left(M_{r} \times r_{k}\right)$ unitary matrix and $\widetilde{\mathbf{R}}_{k}$ is the $r_{k} \times r_{k}$ upper triangular matrix. Let $\mathbf{y}_{k}^{\prime}=\widetilde{\mathbf{Q}}_{k}^{\mathcal{H}} \tilde{\mathbf{y}}_{k}=\widetilde{\mathbf{R}}_{k} \mathbf{s}_{k}$, the interferencefree $\mathbf{s}_{k}\left[r_{k}\right]$ is detected first, and then its contribution can be removed when detecting $\mathbf{s}_{k}\left[r_{k}-1\right]$. The process continues until all $\mathbf{s}_{k}$ elements are detected. By rearranging the columns of $\widetilde{\mathbf{H}}_{k}$ firstly according to SNR (and the corresponding $\mathbf{s}_{k}$ elements), the performance can be further improved at the cost of sorting effort [18].

\section{HCDD Transceiver Architecture and its Application in SVB}

In mobile communication systems, video streaming applications have been increasing rapidly and thus demanding more bandwidth. Therefore, it is important to develop bandwidth-efficient video transmission techniques. By broadcasting/multicasting, multiple mobile terminals can be served with common data and radio resources. Compared to unicasting that serves each terminal with individual data and consumes multiple radio resources, broadcasting/multicasting is much more efficient in bandwidth utilization. However, unlike the unicasting case that each terminal can be served individually, in the case of broadcasting/multicasting all terminals are served with common transmission parameters. Thus, it is challenging to assure that most of the serving terminals

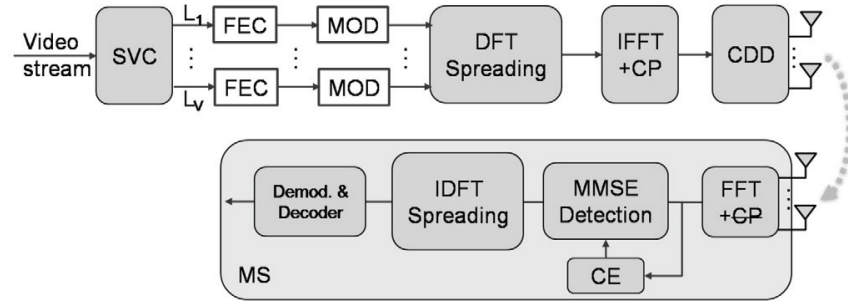

Fig. 2. Existing SCFDM-SVB transmitter and receivers.

can satisfy their services with variable channel conditions and other factors .

Scalable video coding (SVC), an extension of the H.264/AVC video compression standard [19], is a promising solution. SVC can separate a high-quality video stream into several distinct streams, namely the basic layer and advanced layer(s). As long as the basic layer can be decoded correctly, the terminal can at least display a low resolution video. The display quality can be enhanced if the next advanced layer is also decoded correctly. When all layers are decoded successfully, the original high-quality video is recovered. With this scalability, terminals with different channel effects and form factors can be served with different video quality achieved.

Since the basic layer in SVC is the most fundamental, it should be transmitted with more reliable transmission parameters. In the existing literature, different modulation \& coding schemes (MCS) and power levels are allocated to different layers for different levels of protection, such as single-carrier frequency division multiplexing (SCFDM)-based SVB [20], as illustrated in Fig. 2. On the other hand, the proposed HCDD scheme can provide another dimension of protection. With HCDD, different layers can be transmitted with different diversity gain and multiplexing rates. In addition, the flexibility of the HCDD scheme in rate assignment makes it easy to accommodate the SVC layers of arbitrary data rates. The signal processing of HCDD-based SVB is illustrated in Fig. 3.

As for the receivers, with HCDD-based SVB, the smaller terminals with single antenna only need to access the subcarriers carrying the basic layer with highest reliability $\left(r_{k}=1\right)$. In practice, because a small handheld terminal has a small display, low resolution video is sufficient. Moreover, since small terminals are more sensitive to manufacturing cost and power consumption, single antenna/RF chain and lower complexity are preferred. On the other hand, bigger terminals with two or more antennas can access the subcarriers carrying advanced layers with $r_{k} \geq 2$. More advanced signal processing techniques are also applicable to enhance the service quality further. This is reasonable since a bigger terminal with larger display is more likely to have multiple RF chains and afford higher complexity. As a result, a finer video can be achieved when more receiving antennas and channel ranks are available, and basic video is always available for all terminals. Furthermore, small terminals can be made more simply, less expensively, and with better energy efficiency than conventional SCFDM-based SVB receivers.

\section{Numerical Results}

In this section, we compare the performance of the proposed HCDD scheme with current CDD, SCDD, and spatial multi- 


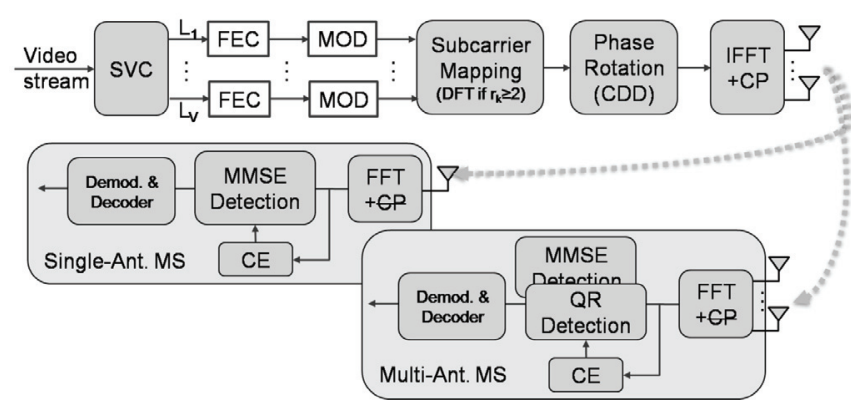

Fig. 3. Proposed HCDD-SVB transmitter and receivers.

plexing schemes. As mentioned above, the major advantage of the HCDD scheme is its flexibility to achieve non-integer diversity-multiplexing-tradeoff requirements. This advantage of the proposed HCDD will be shown in terms of outage capacity and bit error rate.

Without CSI at the transmitter, the per-tone channel capacity for MIMO-OFDM systems is written as [21]

$$
\begin{aligned}
C_{k} & =\log _{2}\left[\operatorname{det}\left(\mathbf{I}_{M_{r}}+\frac{E_{S}}{M_{t} N_{0}} \mathbf{H}_{k} \mathbf{H}_{k}^{\mathcal{H}}\right)\right] \\
& =\sum_{i=1}^{R_{k}} \log _{2}\left(1+\frac{E_{S}}{M_{t} N_{0}} \lambda_{i}\right)
\end{aligned}
$$

where $R_{k}$ is the rank of $\mathbf{H}_{k}$ and $\lambda_{i}\left(i=1,2, \ldots, R_{k}\right)$ are the positive eigenvalues of $\mathbf{H}_{k} \mathbf{H}_{k}^{\mathcal{H}}$. Thus the channel capacity averaged over all subcarriers is given by

$$
C=\frac{1}{N} \sum_{k=1}^{N} C_{k}
$$

The per-tone channel capacities for the HCDD and SCDD schemes are obtained by substituting channel matrix $\widetilde{\mathbf{H}}_{k}$ in (8) and $\mathbf{H}_{k}^{(S)}$ in (4) into (13). The $q \%$ outage capacity is defined as the capacity that $(100-q) \%$ independent realizations can achieve.

\section{A. Outage Capacity Performance}

Figure 4 shows the $1 \%$ outage capacity of the SM, CDD, HCDD schemes in $2 \times 2$ MIMO-OFDM systems, where the multiplexing rates of the SM and CDD schemes are two and one, respectively. In contrast, the multiplexing rate of the HCDD scheme can be flexibly chosen as any non-integer number between one and two. Figure 4 shows that the HCDD with $r_{G}=1.5$ is located exactly between the other two schemes. Suppose that $10 \mathrm{bps} / \mathrm{Hz}$ is required to guarantee $99 \%$ of all channels when $E_{b} / N_{0}$ reaches $25 \mathrm{~dB}$. Then the proposed HCDD scheme with $r_{G}=1.5$ can satisfy this particular requirement.

Figure 5 shows the $1 \%$ outage capacity of various antenna multiplexing and diversity schemes for $4 \times 4$ MIMO-OFDM systems, including HCDD, SM $(r=4)$, SCDD $(r=2)$, and CDD $(r=1)$. The non-integer multiplexing rates for the HCDD scheme are achieved by assigning $\left[\begin{array}{lll}N_{1} & N_{2} & N_{3}\end{array}\right]$ (number of subcarriers with $r_{k}=1, r_{k}=2$, and $r_{k}=4$ in each 10-subcarrier resource block). In this example, the values of [ [ $\left.\begin{array}{lll}7 & 2 & 1\end{array}\right]$ and $\left[\begin{array}{lll}2 & 2 & 6\end{array}\right]$ result in the multiplexing rates of $r_{G}=1.5$ and $r_{G}=3$, respectively. As shown in the figure,

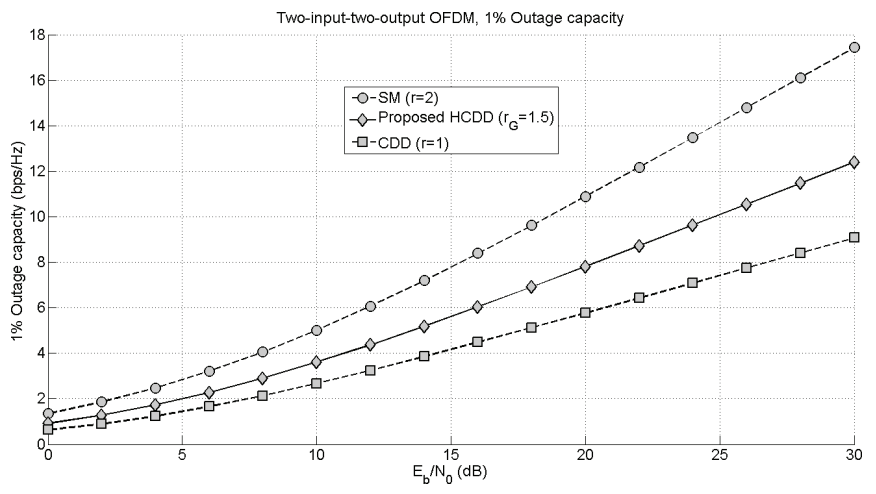

Fig. 4. The $1 \%$ outage capacity performance of different transmission schemes in the $2 \times 2$ MIMO-OFDM systems.

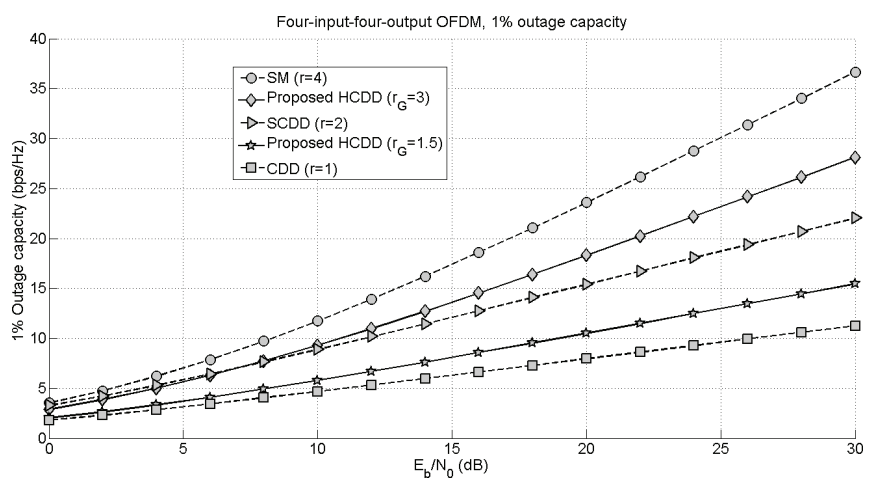

Fig. 5. The $1 \%$ outage capacity performance of different transmission schemes in the $4 \times 4$ MIMO-OFDM systems.

the HCDD scheme can fill the performance gap between the existing antenna diversity and multiplexing schemes, thereby providing a wider range of non-integer multiplexing rates.

Figure 6 shows the $1 \%$ outage capacity of various antenna multiplexing and diversity schemes for $4 \times 4$ MIMO-OFDM systems with the same rate $(r=2)$. MMSE detection is applied for DSTTD transmission [22]. SCDD can be regarded as one special case of HCDD, where all subcarriers are assigned the same rate as two. The same target rate can also be implemented by other subcarrier-rate combinations of the HCDD scheme. Though DSTTD has similar outage capacity, the CDD-like schemes (including SCDD and HCDD) are favored in terms of lower complexity, less stringent constraints on channel coherence, and more flexibility on antenna numbers [16]. It can be observed that if more subcarriers are assigned high rate, the outage capacity is slightly degraded due to increased spatial interference in high-rate subcarriers.

\section{B. Impact of Antenna Correlation}

In Figs. 4 and 5, it is assumed that the correlation among transmitting antennas is kept as small as 0.1. The correlated channel can be described as

$$
\mathbf{H}_{k}=\mathbf{H}_{k}^{(w)} \mathbf{R}^{\frac{1}{2}},
$$

where $\mathbf{H}_{k}^{(w)}$ is the uncorrelated channel matrix; $\mathbf{R}$ is the exponential correlation matrix, $\mathbf{R}_{i, j}=\rho^{|i-j|}$; and $\rho$ is the correlation coefficient between consecutive antennas. In [23], high antenna correlation will decrease the received SNR. 


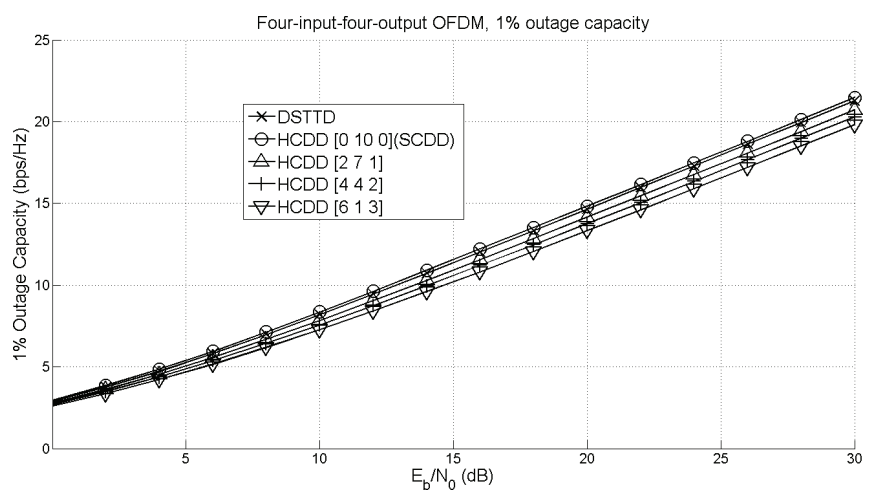

Fig. 6. The $1 \%$ outage capacity performance of different transmission schemes with the same multiplexing rate in the $4 \times 4$ MIMO-OFDM systems.

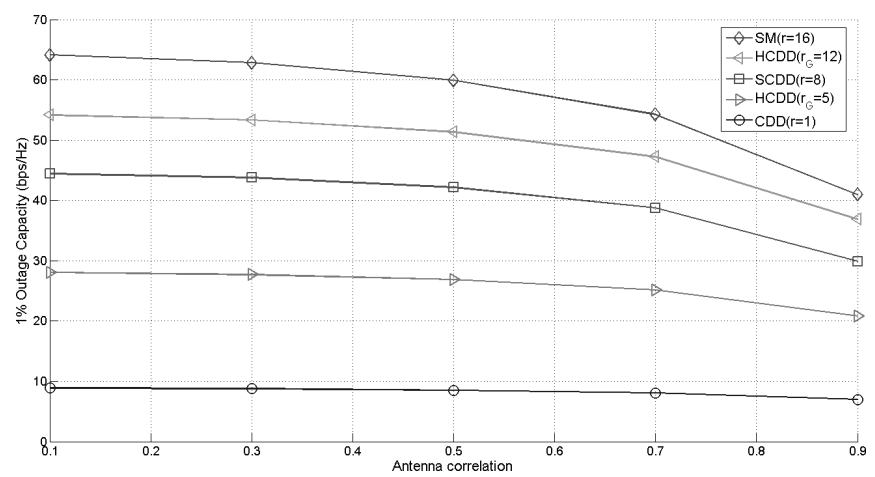

Fig. 7. The $1 \%$ outage capacity performance in the $16 \times 16$ MIMO-OFDM systems with $E_{S} / N_{0} 15 \mathrm{~dB}$ and different transmitting antenna correlations.

However, the effects of antenna correlation are different in various transmission schemes. For the SM-based MIMO systems, the ergodic channel capacity is upper-bounded by $\log _{2} \operatorname{det}\left(\mathbf{I}_{M_{r}}+\frac{E_{S}}{M_{t} N_{0}} \mathbf{R}\right)$ [23]. For CDD, the upper bound of the ergodic channel capacity at subcarrier $k$ is expressed as

$$
\log _{2} \operatorname{det}\left(\mathbf{I}_{M_{r}}+\frac{E_{S}}{M_{t} N_{0}} \mathbf{c}_{k}^{\mathcal{H}} \mathbf{R} \mathbf{c}_{k} \mathbf{I}_{M_{r}}\right)
$$

where $\mathbf{c}_{k}=\left[\begin{array}{lll}W_{N}^{\delta_{1} k} & \ldots & W_{N}^{\delta_{M_{t}} k}\end{array}\right]^{T}[24]$.

Figure 7 shows the $1 \%$ outage capacity of $16 \times 16$ MIMOOFDM systems versus antenna correlation with fixed $E_{S} / N_{0}$ $(15 \mathrm{~dB})$. The outage capacity of every scheme is degraded when channel correlation increases. However, the capacity of SM drops by $36 \%(64.14 \mathrm{bps} / \mathrm{Hz}$ to $40.99 \mathrm{bps} / \mathrm{Hz})$ when channel correlation increases from 0.1 to 0.9 . By contrast, the capacity of CDD drops by $21 \%(8.874 \mathrm{bps} / \mathrm{Hz}$ to 7.006 $\mathrm{bps} / \mathrm{Hz}$ ) when antenna correlation increases from 0.1 to 0.9 . As antenna correlation increases from 0.1 to 0.9 , the capacity of the proposed HCDD decreases by $32 \%$ and $26 \%$ when the rate is 12 and 5 respectively, which are between the SM and CDD schemes.

\section{Bit Error Rate}

Here we show the bit error rate performance of different antenna and multiplexing schemes in MIMO-OFDM systems. Table I lists the system parameters used in our simulations. Figure 8 shows the bit error rate (BER) performance of the HCDD scheme with the multiplexing rate $r_{G}=1.25$ and
TABLE I

Bit ERror Rate Simulation PARAMETERS

\begin{tabular}{|c|c|}
\hline Parameter & Description \\
\hline Number of subcarriers $(N)$ & 128 \\
Carrier bandwidth & $10 \mathrm{MHz}$ \\
Cyclic prefix length & $N / 4$ \\
Channel Model & Flat-fading channel \\
Channel Code & Convolutional encoder, \\
& Vitervi decoder. \\
Constraint length & $7,[133171]$. \\
Modulation & BPSK \\
MIMO Receiver & Linear MMSE \\
Channel Estimation & Ideal Channel Estimation \\
\hline
\end{tabular}

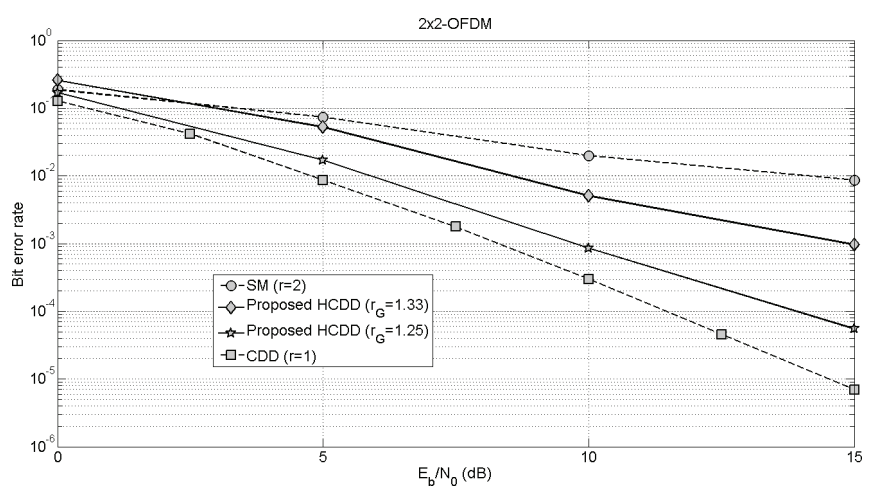

Fig. 8. Comparison of bit error rate performance of SM, HCDD, and CDD antenna transmission schemes in the $2 \times 2$ MIMO-OFDM systems.

$r_{G}=1.33$. For comparison, the BER performances of the MIMO-OFDM systems with SM and CDD are also shown. It is well known that the diversity gain can be estimated from the slope of the BER curve against $E_{b} / N_{0}$. From Fig. 8 , it can be seen that the SM MIMO systems and CDD MIMO systems can be viewed as the extreme cases of the HCDD scheme. In the considered case, the multiplexing rates of SM MIMO systems and CDD MIMO systems are 2 and 1, respectively. The multiplexing rates of the HCDD scheme are 1.33 and 1.25 and can be any non-integer between 1 and 2, thereby providing more flexibility in system design.

Figure 9 shows the BER performance of the HCDD scheme with rate $r_{G}=2.5$ and $r_{G}=3$ in the $4 \times 4$ MIMO-OFDM systems. Compared to the SM MIMO systems $(r=4)$ and the SCDD $(r=2)$ schemes, the ability to choose multiplexing rates in the proposed HCDD MIMO-OFDM systems can provide more flexibility in the system design. For the 0.01 BER requirement, the proposed scheme can achieve multiplexing rates from 2 up to 4 by adjusting the values of $\left[\begin{array}{lll}N_{1} & N_{2} & N_{3}\end{array}\right]$.

Figure 10 shows the multiplexing rates vs. BER for HCDD, SCDD, and SM, etc., which is obtained by observing the results in Figs. 8 and 9. According to Figs. 8 and 9, we obtain BER at the $y$-axis for a fixed $E_{b} / N_{0}$ at $x$-axis. As shown in the figure, one can see that the proposed HCDD fills the gap of the CDD/SCDD and SM for the cases of $2 \times 2$ and $4 \times 4$ MIMO-OFDM at $E_{b} / N_{0}=10 \mathrm{~dB}$.

\section{Simulation Results of $S V B$ using $H C D D$}

Three layers are transmitted by a four-antenna BS. For each OFDM symbol, the data bits of each layer is 160,160 , and 240 bits, respectively. Layer 1 is the basic layer, and the other two layers are advanced layers. Layer 2 can be decoded only 


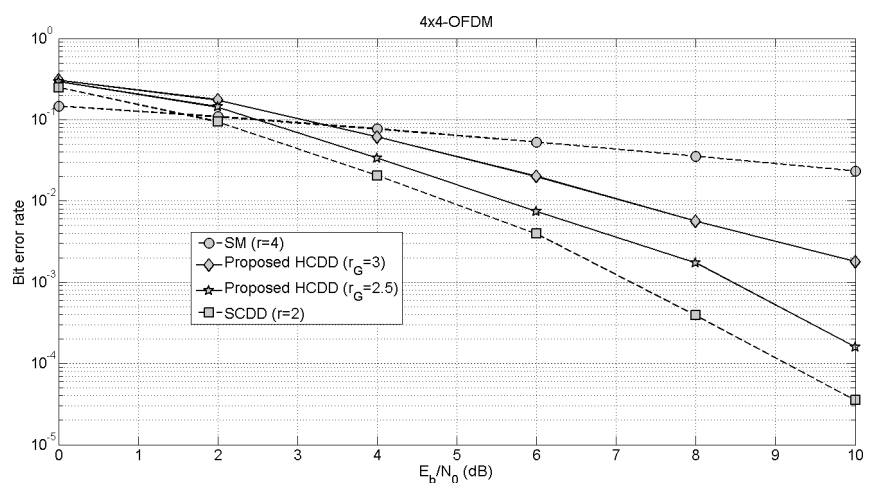

Fig. 9. Comparison of the bit error rate performance of HCDD, CDD, and SM antenna transmission schemes in the $4 \times 4$ MIMO-OFDM systems.

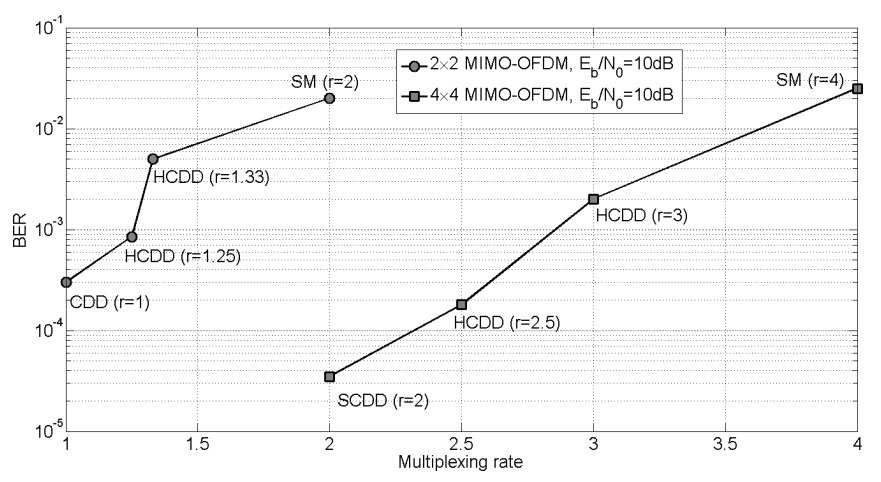

Fig. 10. Multiplexing rate vs. BER of HCDD, CDD, and SM schemes for $2 \times 2$ and $4 \times 4$ MIMO-OFDM at $E_{b} / N_{0}=10 \mathrm{~dB}$.

if layer 1 is decoded successfully. Layer 3 can be decoded only if layer 1 and 2 are both decoded successfully. Table II lists the simulation parameters .

Figure 11 (a) shows the coverage of the basic layer for receivers with a single antenna $\left(M_{r}=1\right)$. Though HCDD is slightly inferior in basic layer coverage, the HCDD receivers have the advantage of less complexity than SCFDM receivers. The performance with HCDD can be improved by adjusting transmission power or cell sizes. Figure 11 (b) shows the coverage results for $M_{r}=4$. All layers can be detected, and HCDD is slightly superior to SCFDM in all layer coverage. In addition to the comparable coverage performance, HCDD receivers still have the advantage of less complexity than SCFDM receivers.

Complexity is another important factor in receiver design, and it can be measured by the number of floating point operations (flops). The main difference between HCDD- and SCFDM-based receivers lies in the detection method and inverse DFT (IDFT) spreading. For SCFDM receivers, MMSE detection and IDFT spreading takes $N\left(4 M_{r}+1\right)$ and $2 N^{2}-N$ flops, respectively. Note that $N$, the subcarrier number, is much larger than $M_{r}$, the receive antenna number. For HCDD receivers, MMSE detection (at subcarries $r_{k}=1$ ) and QRD require $4 M_{r}+1$ and $3 r_{k}^{2}\left(M_{r}-r_{k} / 3\right)$ [25] flops per subcarrier, respectively. It can be observed that for SCFDM receivers, no matter how many antennas are used, MMSE is applied to every data subcarrier, and IDFT spreading takes $O\left(N^{2}\right)$ flops. On the contrary, for HCDD receivers, MMSE or QRD is applied only to the subcarriers the receiver can detect according to
TABLE II

LiNK LEVEL SiMULATION PARAMETERS

\begin{tabular}{|c|c|}
\hline Parameter & Value \\
\hline Channel model & ITU-R VA \\
\hline Bandwidth & $5 \mathrm{M}$ \\
\hline No. of Carriers $(N)$ & 512 \\
\hline Cell radius & $500 \mathrm{~m}$ \\
\hline Transmission Power & $30 \mathrm{dBm}$ \\
\hline Path loss & $128.1+37.6 \log _{10}(D)(\mathrm{D}: \mathrm{km})$ \\
\hline Noise PSD & $-174 \mathrm{dBm} / \mathrm{Hz}$ \\
\hline No. of User Terminals & 1000 \\
\hline
\end{tabular}

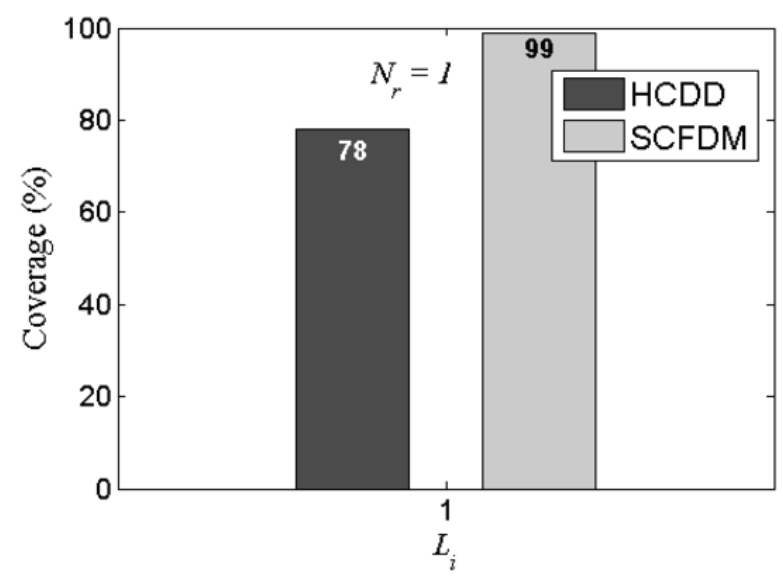

(a)

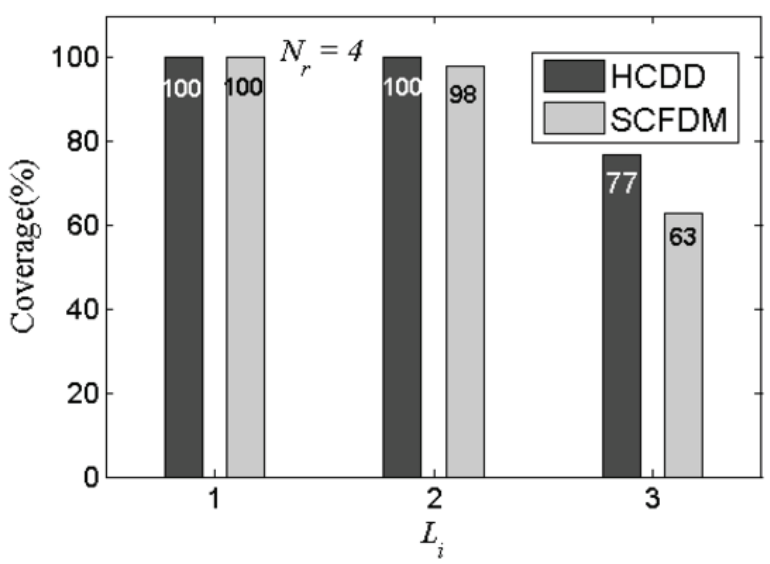

(b)

Fig. 11. Coverage of SVB layers for receivers with (a) $M_{r}=1$ and (b) $M_{r}=4$.

the antenna number. Furthermore, HCDD receivers are free from the $O\left(N^{2}\right)$ flops inherent in SCFDM receivers. The complexity reduction is a significant advantage of HCDD over conventional SCFDM schemes.

\section{CONCLUding Remarks}

This paper proposes a novel HCDD architecture to achieve flexible diversity-multiplexing tradeoff in MIMO-OFDM systems. Unlike the existing transmitting antenna diversity scheme, which is suitable only for integer multiplexing rates, the proposed HCDD MIMO-OFDM scheme can achieve noninteger multiplexing rates by taking advantage of the rate assignment in the degree of freedom - subcarrier. Our simu- 
lation results show that the proposed HCDD can successfully fill the performance gap between the existing MIMO schemes, which can only provide the integer-value multiplexing rates. The idea of utilizing the dimension - subcarrier of OFDM - to achieve the non-integer multiplexing rates can achieve the diversity and multiplexing tradeoff with more flexibility and can provide important insights into the design of future MIMO-OFDM systems. The combination of HCDD with SVB provides flexible diversity gains to different layers, and the overall receiver complexity is lower than conventional scheme.

\section{REFERENCES}

[1] L. Zheng and D. Tse, "Diversity and multiplexing: a fundamental tradeoff in multiple-antenna channels," IEEE Trans. Inf. Theory, vol. 49, no. 5, pp. 1073-1096, May 2003.

[2] T. Hwang, C. Yang, G. Wu, S. Li, and G. Ye Li, "OFDM and its wireless applications: a survey," IEEE Trans. Veh. Technol., vol. 58, no. 4, pp. 1673-1694, May 2009.

[3] Q. Li, G. Li, W. Lee, M.-I. Lee, D. Mazzarese, B. Clerckx, and Z. Li, "MIMO techniques in WiMAX and LTE: a feature overview," IEEE Commun. Mag., vol. 48, no. 5, pp. 86-92, May 2010.

[4] Y. Li, J. H. Winters, and N. R. Sollenberger, "MIMO-OFDM for wireless communications: signal detection with enhanced channel estimation," IEEE Trans. Commun., vol. 17, no. 9, pp. 1233-1477, Sep. 2002.

[5] L. Zhao and V. Dubey, "Detection schemes for space-time block code and spatial multiplexing combined system," IEEE Commun. Lett., vol. 9, no. 1 , pp. 49-51, 2005.

[6] M. B. Obando, W. C. Freitas Jr., and F. R. P. Cavalcanti, "Switching between hybrid MIMO structures for video transmission based on distortion model," in Proc. 2010 IEEE Veh. Technol. Conf. - Fall.

[7] J. T. Wang, "Combined diversity and multiplexing for multiple-input multiple-output system with cochannel interference," IET Commun., vol. 4, no. 10, pp. 1164-1173, 2010.

[8] Y. Lee and H.-wei Shieh, "A simple layered space-time block nulling technique for DSTTD systems," IEEE Commun. Lett., vol. 3, no. 8, pp. 4-6, 2011.

[9] A. Dammann and S. Kaiser, "Performance of low complex antenna diversity techniques for mobile OFDM systems," in Proc. 2001 Int. Workshop Multi-Carrier Spread-Spectrum Related Topics, pp. 53-64.

[10] A. Sezgin, M. Charafeddine, S. Pereira, and A. Paulraj, "Diversitymultiplexing tradeoff of stacked cyclic delay diversity schemes," in Proc. 2007 Allerton Conf. Commun., Contr., Computing.

[11] M. I. Rahman, S. S. Das, E. de Carvalho, and R. Prasad, "Spatial multiplexing in OFDM systems with cyclic delay diversity," in Proc. 2007 IEEE Veh. Technol. Conf. - Spring, pp. 1491-1495.

[12] 3GPP TS 36.211 V10.4.0, "Evolved universal terrestrial radio access (EUTRA); Physical channels and modulation (Release 10)," Dec. 2011.

[13] D. Ballesteros, H.-W. Chang, and L.-C. Wang, "On the performance of hybrid stacked cyclic delay diversity schemes in MIMO-OFDM," in Proc. 2010 IEEE Int. Conf. Commun.

[14] M. Bossert, A. Huebner, F. Schuehlein, H. Haas, and E. Costa, "On cyclic delay diversity in OFDM based transmission schemes," in Proc. 2002 OFDM Workshop.

[15] Y. J. Kim, H. Y. Kim, M. Rim, and D. W. Lim, "On the optimal cyclic delay value in cyclic delay diversity," IEEE Trans. Broadcast., vol. 55, no. 4 , pp. $790-795$, Dec. 2009.

[16] G. Bauch and J. Malik, "Cyclic delay diversity with bit-interleaved coded modulation in orthogonal frequency division multiple access," IEEE Trans. Wireless Commun., vol. 5, no. 8, pp. 2092-2100, Aug. 2006.

[17] P. Sklikas, M. Mjeku, and N. Gomes, "Mac layer performance evaluation of IEEE 802.16e radio-over-fiber networks," in Proc. 2010 IEEE Topical Meeting Microwave Photonics, pp. 350-353.

[18] D. Wubben, R. Bohnke, V. Kuhn, and K.-D. Kammeyer, "MMSE extension of V-BLAST based on sorted QR decomposition," in Proc. 2003 IEEE Veh. Technol. Conf. - Fall, vol. 1, pp. 508-512.
[19] H. Schwarz, D. Marpe, and T. Wiegand, "Overview of the scalable video coding extension of the H.264/AVC standard," IEEE Trans. Circuits Syst. Video Technol., vol. 17, no. 9, pp. 1103-1120, Sep. 2007.

[20] S. Jaeckel and V. Jungnickel, "Scalable video transmission in multiantenna broadcast systems," Proc. 2008 European Wireless Conf.

[21] A. Paulraj, R. Nabar, and D. Gore, Introduction to Space-Time Wireless Communications. Cambridge University Press, 2003.

[22] H.-W. Chang, L.-C. Wang, and Z.-H. Chou "Macrodiversity antenna combining for MIMO-OFDM cellular mobile networks in supporting multicast traffic," in Proc. 2011 IEEE Veh. Technol. Conf. - Spring, 2011.

[23] S. Loyka, "Channel capacity of MIMO architecture using the exponential correlation matrix," IEEE Commun. Lett., vol. 5, no. 9, pp. 369-371, 2001.

[24] A. Sadeque and M. Saquib, "Pseudo random binary phase offset scheme for multi-carrier delay diversity modulation," IEEE Trans. Wireless Commun., vol. 10, no. 5, pp. 1495-1503, May 2011.

[25] G. H. Golub and C. F. V. Loan, Matrix Computations, 3rd edition. The Johns Hopkins University Press, 1989.

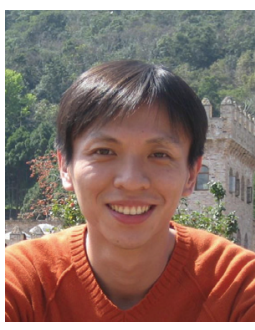

Hsien-Wen Chang (S'09-M'12) received the B.S. and M.S. degrees in electrical engineering from National Tsing Hua University, Hsin-chu, Taiwan, in 1999 and 2001, respectively. From 2001 to 2005, he was with the National Space Organization. Since 2006, he has joined the Information and Communications Research Laboratories in the Industrial Technology Research Institute. He is currently working toward his $\mathrm{Ph} . \mathrm{D}$. degree at the Institute of Communications Engineering, National Chiao Tung University. His research interests include MIMOOFDM systems, digital broadcasting, and radio resource allocation in wireless networks. He has published several international conference papers and holds several patents in the United States, Mainland China, and Taiwan, R.O.C.

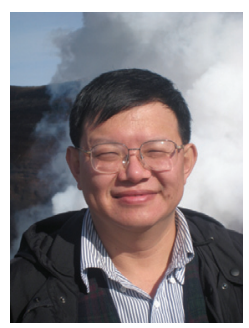

Li-Chun Wang (M'96-SM'06-F'11) received the B.S. degree from National Chiao Tung University, Taiwan, R. O. C., in 1986, the M.S. degree from National Taiwan University in 1988, and the Ms.Sci. and $\mathrm{Ph} . \mathrm{D}$. degrees from the Georgia Institute of Technology, Atlanta, in 1995, and 1996, respectively, all in electrical engineering.

From 1990 to 1992, he was with the Telecommunications Laboratories of the Ministry of Transportation and Communications in Taiwan (currently the Telecom Labs of Chunghwa Telecom Co.). In 1995, he was affiliated with Bell Northern Research of Northern Telecom, Inc., Richardson, TX. From 1996 to 2000, he was with AT\&T Laboratories, where he was a Senior Technical Staff Member in the Wireless Communications Research Department. Since August 2000, he has joined the Department of Electrical and Computer Engineering of National Chiao Tung University in Taiwan and is the current Chairman of the same department. His current research interests are in the areas of radio resource management, crosslayer optimized techniques for heterogeneous wireless networks, and cloud computing for mobile applications.

Dr. Wang was elected to the IEEE Fellow grade in 2011 for his contributions in cellular architecture and radio resource management in wireless networks. He won the Distinguished Research Award of the National Science Council, Taiwan, in 2012, and was a co-recipient (with Gordon L. Stüber and Chin-Tau Lea) of the 1997 IEEE Jack Neubauer Best Paper Award for his paper "Architecture Design, Frequency Planning, and Performance Analysis for a Microcell/Macrocell Overlaying System," IEEE TRANSACTIONS ON Vehicular TeChNOLOgY, vol. 46, no. 4, pp. 836-848, 1997. He has published over 150 journal and international conference papers. He served as an Associate Editor for the IEEE TRANSACTIONS ON WIRELESS COMMUNICATIONS from 2001 to 2005, the Guest Editor of the Special Issue on "Mobile Computing and Networking" for the IEEE Journal ON SELECTED AREAS IN COMMUNICATIONS in 2005 and on "Radio Resource Management and Protocol Engineering in Future IEEE Broadband Networks" for IEEE Wireless Communications Magazine in 2006. He holds 10 US patents. 